\title{
Thermoelectric properties and intrinsic conduction processes in DBSA and NaSIPA doped polyanilines
}

Laura Horta-Romarís ${ }^{1}$, M. Victoria González-Rodríguez ${ }^{1}$, Aurora Lasagabáster ${ }^{1,2}$, Francisco Rivadulla ${ }^{3}$, María-José Abad*1

${ }^{1}$ Universidade da Coruña Grupo de Polímeros, Centro de Investigacións Tecnolóxicas, Campus de Esteiro, 15471 Ferrol, Spain.

${ }^{2}$ Dpto Química Orgánica I, Facultad de Óptica, Universidad Complutense de Madrid, Arcos de Jalón 118, Madrid 28037, Spain.

${ }^{3}$ Centro de Investigación en Química Biolóxica e Materiais Moleculares, Campus Vida, Universidade de Santiago de Compostela, 15782-Santiago de Compostela, Spain.

*Corresponding Author e-mail: mjabad@udc.es.

\begin{abstract}
Seeking to gain fundamental understanding of the thermoelectric (TE) behavior of polyanilines (PANIs), structure- property relationships of PANI nanorods, doped with dodecylbenzenesulfonic acid (DBSA) and 5-sulfoisophtalic acid sodium salt (NaSIPA), and prepared by an indirect synthetic route, are discussed in terms of the contribution of the acid concentrations on the thermoelectric properties.

The synergistic combination of high doping level and layer structure, accounts for the moderately high electrical conductivities $(\sigma)$ and low constant Seebeck coefficients $(\alpha)$ of PANI-DBSA. Conversely, the poor doping ability of NaSIPA and low crystallinity degree explain the low electrical conductivities along with significant increases in Seebeck coefficient values. In relation to conduction mechanisms, PANI-DBSA shows a hopping behavior with a carrier concentration of $c \approx 0.49$ (hole type), while PANINaSIPA displays a diffusive regime, characteristic of degenerate metallic semiconductors, with an estimated charge carrier density of $n \approx 3 \times 10^{21} \mathrm{e} / \mathrm{cm}^{3}$.
\end{abstract}

\section{KEYWORDS}

Conducting polymers, polyaniline, electrical conductivity, Seebeck coefficient, thermoelectrical properties. 


\section{INTRODUCTION}

The organic thermoelectric (TE) materials, as intrinsically conducting polymers (ICPs), are interesting candidates for green thermoelectric energy conversion applications, given their low cost and environmental safety [1-3]. The energy-conversion efficiency of a TE device is a function of the material's figure-of merit (ZT), average working temperature of the device and temperature difference between the hot and cold ends [4]. ZT is directly proportional to the electrical conductivity $(\sigma)$ and the Seebeck coefficient $(\alpha)$ and inversely proportional to the thermal conductivity $(\kappa)[1,2,4-6]$.

$$
Z T=\frac{\sigma \alpha^{2}}{\kappa} T
$$

Thus, a good thermoelectrical material should have high $\sigma$ and $\alpha$, but poor $\kappa$. The three thermoelectrical parameters are interdependent in bulk materials and decoupling these parameters is definitely non-trivial [7].

Polyaniline (PANI) as one of the important ICPs has caused a lot of scientists' interests due to high stability, facile synthesis and tunable electronic properties [8]. Electrical conductivity of PANI increases with doping, which may be achieved by an acid-base reaction. As a result of the protonation of the nitrogen sites in the emeraldine base (EB), the cation radical of one nitrogen acts as a polaronic hole and these holes are charge carriers (p-type doped PANI) [9] (Fig. 1). Electrical conductivity occurs via interpolaron hopping along and across polymer chains [10, 11]. Therefore, the carrier density depends on the degree of protonation or doping level [4, 12]. Furthermore, in most cases PANIs appear to be amorphous, sometimes with some degree of crystallinity, thus heterogeneous conduction is set up involving "islands" of higher conductivity regions separated by lower conductivity regions. The polaron structures are responsible for electrical conduction through the hopping mechanism in the crystalline region [9], while intergrain resonance tunnelling occurs through the strongly localized states in the amorphous media [13]. These systems are featured with low charge carrier mobility and low electrical conductivities, even at high carrier densities [14, 15]. This mixture of metallic and non-metallic behaviour is a key characteristic of all ICPs, including PANI [16].

By contrast, the Seebeck coefficient is more complex and difficult to predict. The reduction of the total number of charge carriers increase the Seebeck coefficient while lowering the electrical conductivity, but because the power factor (PF) scales with $\alpha^{2}$, a 
net increase in the PF can be achieved for certain doping ranges. The identification of conditions conducive to decouple $\sigma$ and $\alpha$, remains challenging [7].

In this context, the control of morphology to obtain nanostructured polymers has proved to be a successful strategy to improve the TE properties of ICPs. Previous works have shown that polyanilines synthesized with nanorod or nanofiber morphology have lower electrical resistance than those synthesized with micrometric size [17-19]. This nanostructuring strategy can increase the Seebeck coefficient, due to improved charge carrier mobility, and also reduce the thermal conductivity [8, 17], resulting in an enhanced ZT.

Specifically, in a preliminary study we have introduced an ecofriendly and scalable indirect synthetic route (dedoping-redoping) [18], leading to organic doped PANIs with nanostructured morphology using either dodecylbenzensulfonic acid (DBSA) or 5sulfoisophtalic acid sodium salt (NaSIPA) as dopants. Both bulky organosulfonic acids maintained a reasonable balance between conductivity, thermal stability and processability which are essential properties for widening industrial applications. In addition, the different chemical structures between the two dopants (rigid NaSIPA in contrast with the long alkyl flexible chains of DBSA, which in addition acts as a plasticizer) led to PANI salts with morphological and structural differences that are considered a good starting point to ascertain structure - properties relations. Since tuning the degree of doping and morphology are crucial for the optimization of thermoelectric performance of ICPs [7], the present work systematically studies the influence of the doping agent and molar acid concentration on the structure, physicochemical and TE properties of PANI-DBSA and PANI-NaSIPA, and seek to shed light on the conduction mechanisms. To the best of our knowledge, there are few studies concerning the TE performance of PANI DBSA [2] and none of PANI NaSIPA. The different nature and doping ability of the two organosulfonic acids lead to dissimilar ZT trends with molar acid concentration and different conduction mechanisms.

\section{EXPERIMENTAL}

\subsection{MATERIALS SYNTHESIS}

Aniline and dodecylbenzensulfonic acid (DBSA) $70 \mathrm{wt}$ \% solution in 2-propanol were purchased from Sigma-Aldrich. The 5-sulfoisophtalic acid sodium salt (NaSIPA) 95 
wt\% was obtained from Alfa Aesar. The potassium peroxodisulfate (APS) and acetone was obtained from Scharlau.

Two PANI samples were synthesized by indirect route involving "dedoping-redoping" steps with two organic sulfonic dopants, DBSA and NaSIPA (Fig. 1) [20]. PANI-HCl, prepared as described by Park et al. [21], was dedoped with $1 \mathrm{M} \mathrm{NH} \mathrm{NH}_{3}$ for $2 \mathrm{~h}$ in ultrasonic bath, filtered under vacuum and washed with water until neutral $\mathrm{pH}$. The resultant PANI-base was redoped with increasing molar concentrations of DBSA in acetone or NaSIPA aqueous solutions during $2 \mathrm{~h}$ in ultrasonic bath. The samples were washed with large volumes of acetone to remove excess free acids [20]. Finally, the products were filtered under vacuum and freeze-dried.

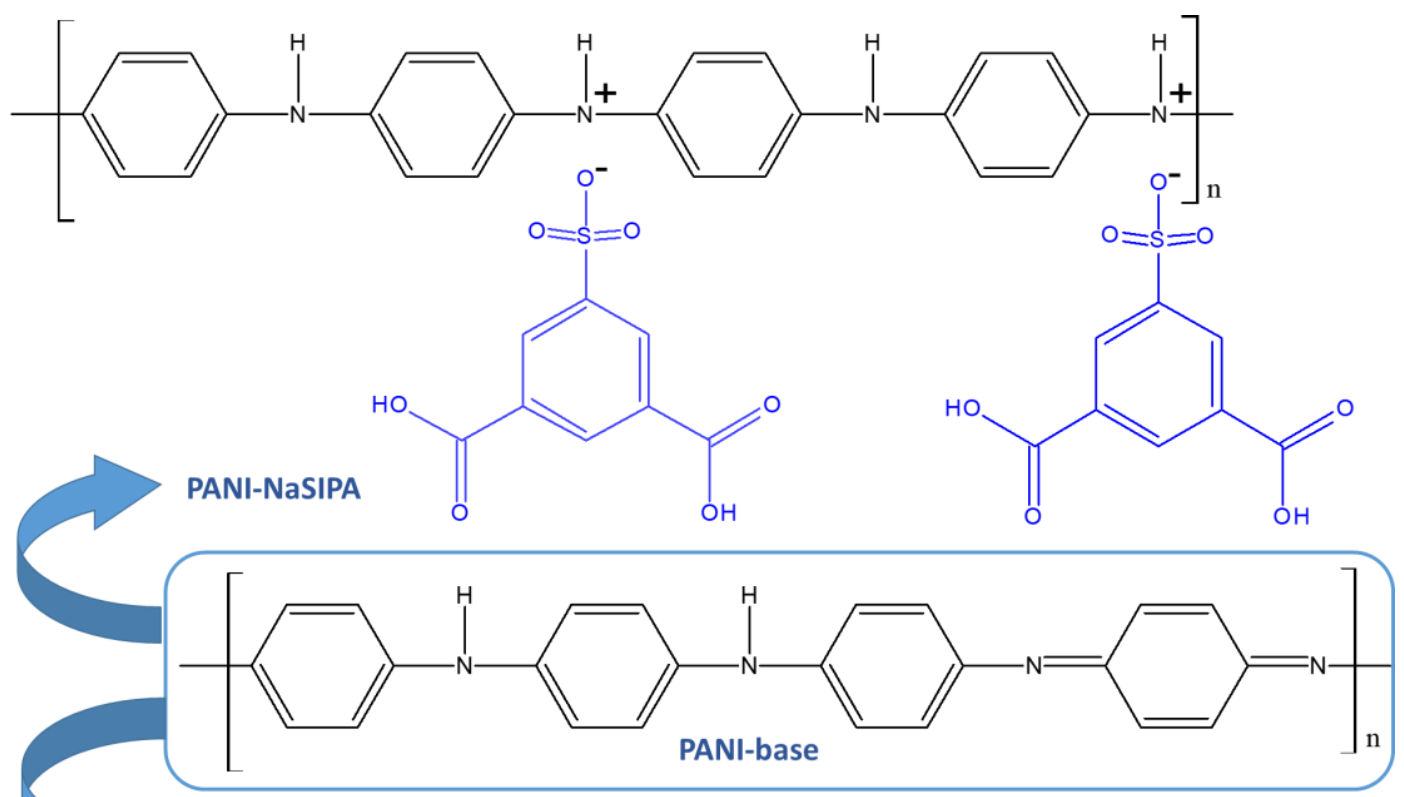

PANI-DBSA

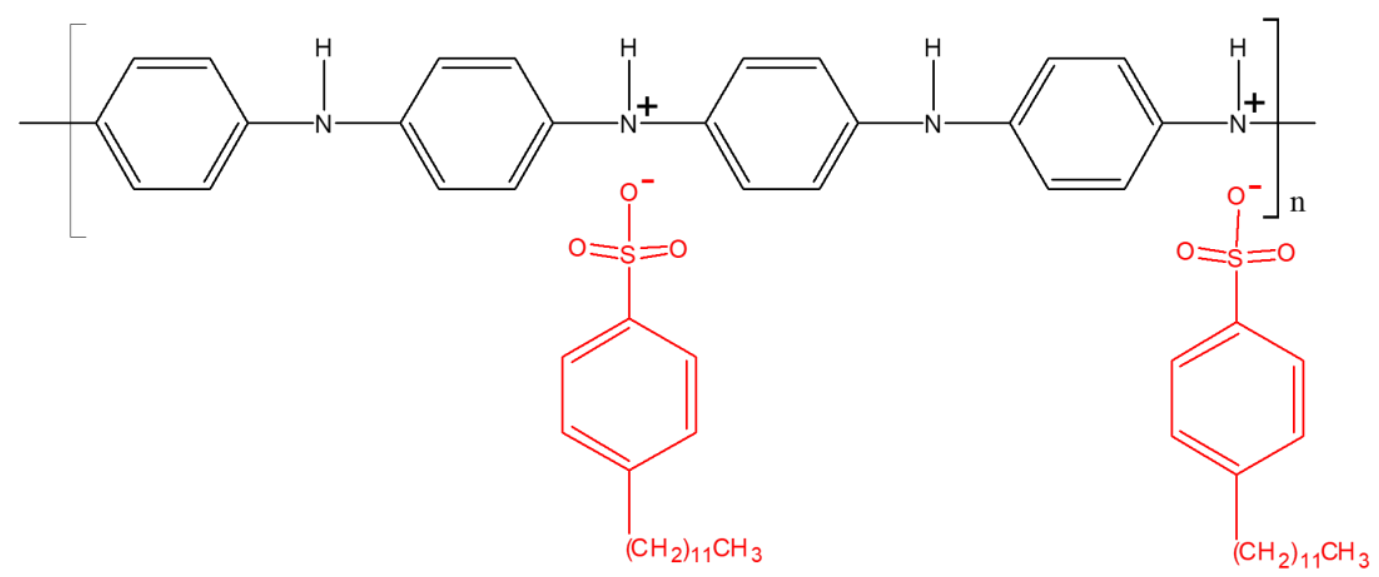

Figure 1. Chemical structure of PANI-base and doped polyanilines, PANI-NaSIPA and PANI-DBSA 
The molecular weight of PANI base is determined from its intrinsic viscosity [ $\eta$, according to the Mark-Houwink-Sakurada equation:

$$
[\eta]=K \cdot M_{\eta}^{\alpha}
$$

where $\mathrm{K}$ and $\alpha$ are constants for a particular polymer-solvent pair at a particular temperature. Poly(p-phenylene terephthalamide) has a rigid chain with conjugated double bonds similar to PANI. Therefore, in a first approximation the values of $\mathrm{K}=$ $1.95 \times 10^{-6}$ and $\alpha=1.36$, characteristic of this polymer in $\mathrm{H}_{2} \mathrm{SO}_{4}$ medium, were used for PANI [22]. The estimated molecular weight of the PANI base is $6,300 \mathrm{Da}\left(\mathrm{R}^{2}=0.97\right)$.

\subsection{CHARACTERIZATION}

The elemental analysis of $\mathrm{C}, \mathrm{H}, \mathrm{N}$, and $\mathrm{S}$ was conducted using ThermoFinnigan FlashEA1112 elemental analyzer.

Fourier transformed infrared (FTIR) spectra of the polymers in $\mathrm{KBr}$ pellets were recorded on a Jasco 4700 spectrometer. The spectra were collected from 4000 to 400 $\mathrm{cm}^{-1}$, with a $4 \mathrm{~cm}^{-1}$ resolution over 100 scans.

X-ray patterns of PANI powders were recorded in step-scan mode from $2^{\circ}$ to $50^{\circ}$ with a $2 \theta$ step of $0.05^{\circ}$ using a D5000 diffractometer (XRD, Siemens- Bruker) with CuK $\alpha$ line irradiation $(\lambda=1.541 \AA$ ). 40 -point smoothing protocol using a Savitzky-Golay filter has been applied to the diffractograms, which were normalized to area 1 (Origin 8.0 graphical software). A split Gaussian function was used to subtract the background and amorphous contributions using the open-source software Fityk. The difference patterns were deconvoluted into the crystalline constituents using Gaussian function peak shape approximation [23] The degree of crystallinity $\left(X_{c}\right)$ was estimated from the percentage of crystalline peak area to total scattered area, the d-spacing was calculated using the Bragg equation, the crystallite domain size (L) was evaluated using the Debye-Scherrer formula from the full width at half-maximum (FWHM)[24].

The morphology of the polymer samples dispersed in isopropanol was evaluated by transmission electron microscopy (TEM, Jeol JEM 1010 (80 KV)).

The three parameters constituting ZT $(\sigma, \alpha$ and $\kappa)$ were measured on specimens prepared by compression molding $(2.5 \times 2.5 \mathrm{~cm} \times 0.5 \mathrm{~mm})$ at $90^{\circ} \mathrm{C}$, applying a pressure of 100 bar for 2 minutes. Electrical resistivity at room temperature was calculated by the four-point method (LORESTA-GP, Mitsubishi Chemical, MCP-T610). The electrical 
conductivities $(\sigma)$ reported for each polymer are the mean values of at least 24 readings determined on different samples.

The Seebeck coefficient $(\alpha)$ was measured using a home-made device (a broad description of the device, experimental parameters and its reproducibility are reported in supplementary material, Fig. S1). The sample was mounted between two copper blocks $\left(4 \times 4 \mathrm{~mm}^{2}\right)$, while a heat pulse was applied to one end of the sample to create a thermal gradient. The total temperature difference was maintained below $2 \mathrm{~K}$, and the linear $\Delta \mathrm{V} / \Delta \mathrm{T}$ variation was recorded at each base temperature (Fig. S2). The measurements were performed under vacuum between 200 and $325 \mathrm{~K}$.

Thermal diffusivities were measured by the flash diffusivity technique with the thermal analyzer (LFA 447 Nanoflash, Germany) on square samples of $8 \mathrm{~mm}$ length and 0.5 $\mathrm{mm}$ thickness at 300,325 and $350 \mathrm{~K}$. Test samples were sprayed with a coating of graphite on both sides before testing (for uniform thermal adsorption). The illumination source was a Xenon flash lamp with a spectral output characterized by a broadband in the visible wavelength and a complex line spectrum in the near IR. Pulse corrected Cape Leman model was used to analyze the data in analysis software. The thermal conductivities were derived from the following equation:

$$
\kappa=a \rho c_{p}
$$

Where $\kappa$ is the thermal conductivity, $a$ is the thermal diffusivity, $\rho$ is the density of samples and $c_{p}$ is the specific heat capacity. Due to the hygroscopic nature of some PANIs the density was estimated from the weight and dimensions of the samples. The specific heat capacity $c_{p}$ of test sample was obtained by Differential Scanning Calorimetry (DSC TA Instruments 2010) using sapphire as standard. Both thermal diffusivity and specific heat capacity are the average value of three replicas for each sample

\section{RESULTS AND DISCUSSION}

When optimizing PANI for thermoelectric applications, the first strategy consists on tuning the doping level, which is controlled by the molar acid concentration in the redoping step.

\subsection{ELECTRICAL CONDUCTIVITY}


Fig. 2 shows the changes in electrical conductivity of PANI salts at room temperature, as a function of DBSA and NaSIPA molar concentrations. The two organic acids lead to dissimilar results; firstly, the room temperature electrical conductivity of PANI-DBSA is always 4 to 70 times higher than that of PANI-NaSIPA. Furthermore, the electrical conductivity of PANI-DBSA increases continuously with the concentration of DBSA up to $\approx 1.25 \mathrm{M}$, reaching relatively large values of $\approx 30 \mathrm{~S} / \mathrm{cm}$, and declines at $1.5 \mathrm{M}$. Regarding PANI-NaSIPA, the electrical conductivity also increases with the concentration of the dopant, although it reaches much smaller values; the maximum value of $\approx 0.5 \mathrm{~S} / \mathrm{cm}$ is achieved at NaSIPA $0.75 \mathrm{M}$.

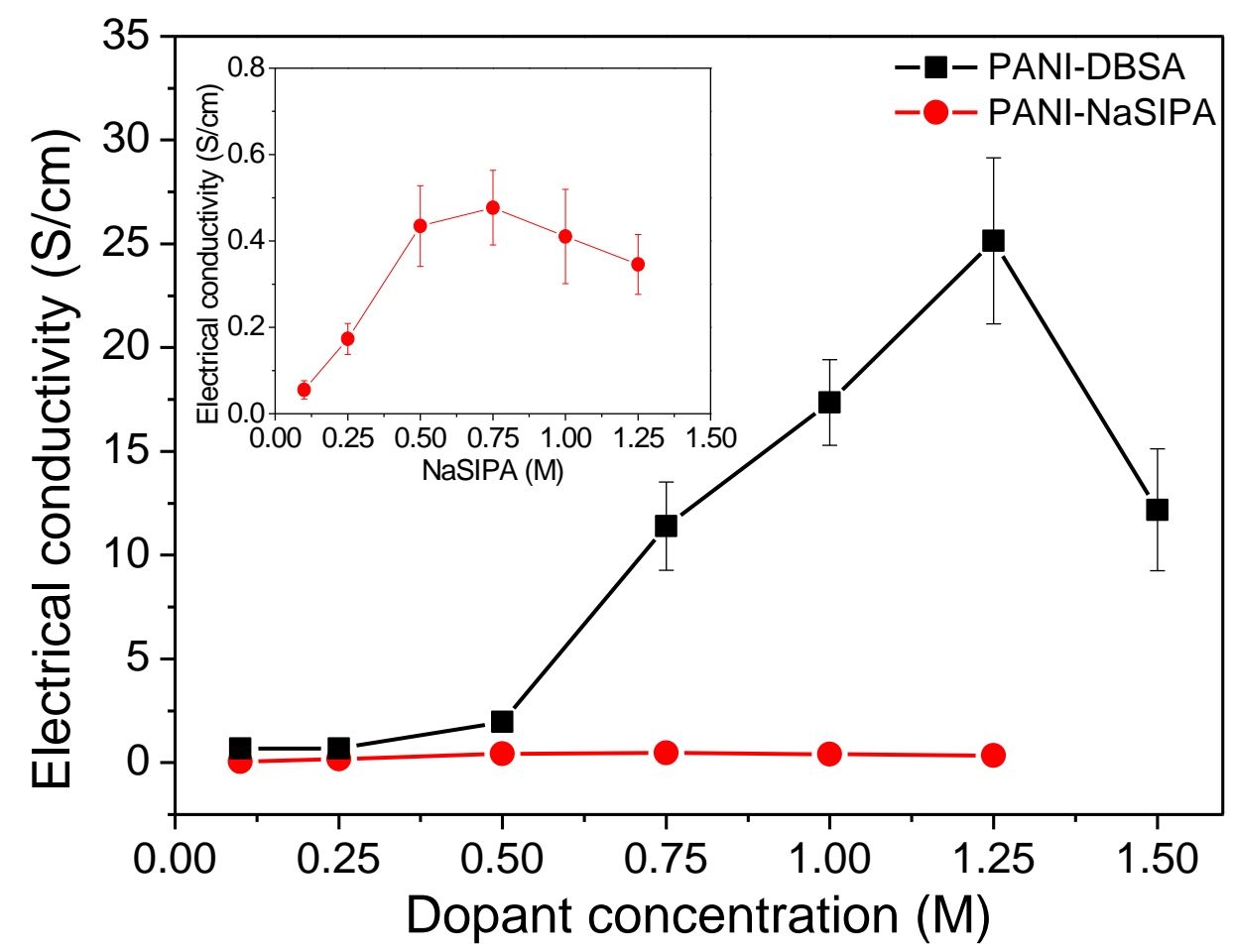

Figure 2. Electrical conductivities of PANI-DBSA and PANI-NaSIPA at various molar acid concentrations at room temperature.

In order to understand the effect of the nature of the organic dopant and its molar concentration on the electrical conductivity of doped-PANIs, the chemical, structural and morphological characterization was addressed by elemental analysis, FTIR, XRD, and TEM.

The S/N ratio calculated by elemental analysis on molar basis has been used to estimate the extent of doping when organic sulfonic acids are used as dopants[18, 25]. For PANIDBSA, the $\mathrm{S} / \mathrm{N}$ ratio gradually rises up to 0.75 for $1.25 \mathrm{M}$ and then declines (Fig. 3). 
This decrease is explained by the surfactant nature of DBSA and the competition effect or molecular attraction between the adjacent DBSA molecules in concentrated $1.5 \mathrm{M}$ solutions, which make it difficult to perform PANI protonation [26]. Moreover, a positive linear correlation between electrical conductivity and doping level has been found (Fig. S3).

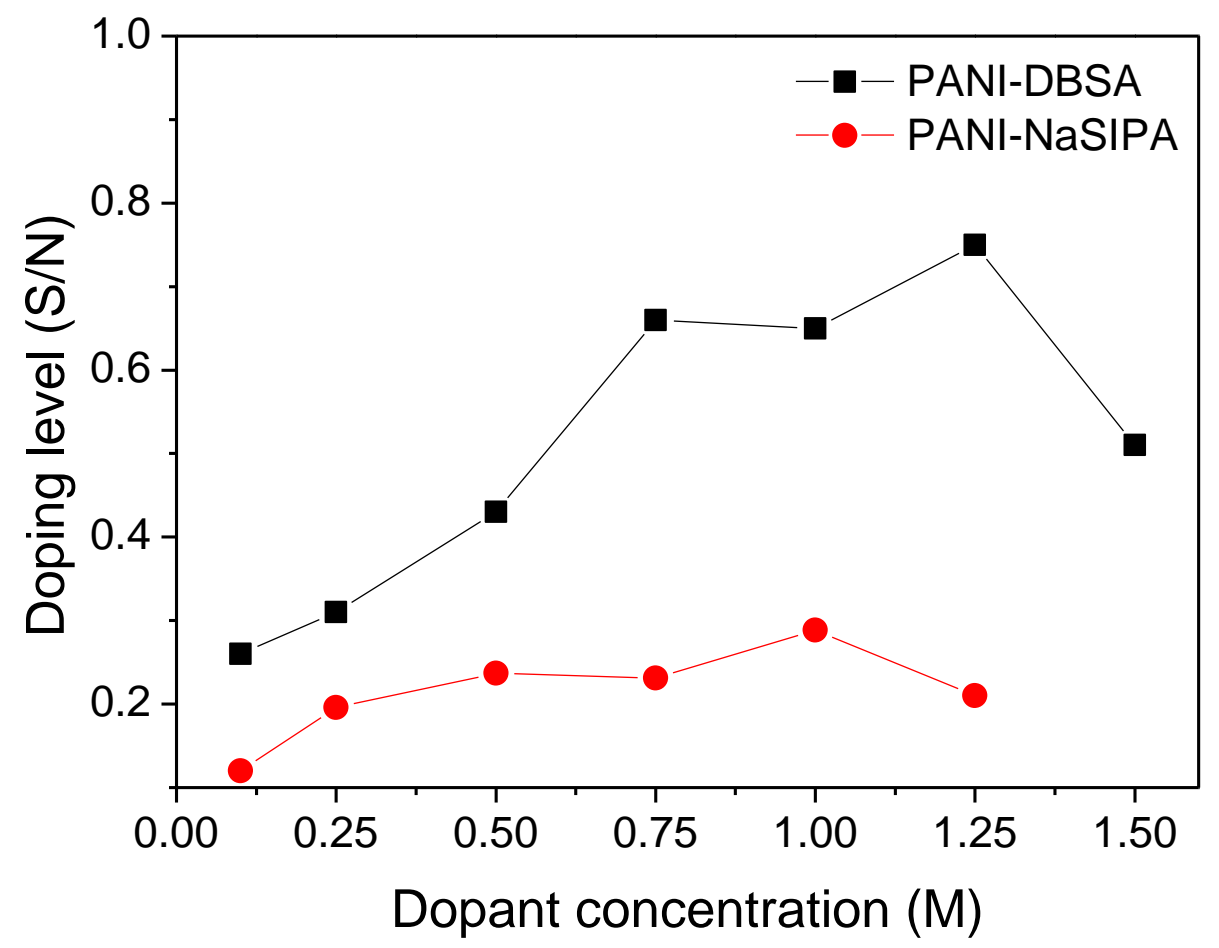

Figure 3. $\mathrm{S} / \mathrm{N}$ ratio calculated by elemental analysis as a function of the molar acid concentration.

By contrast, the effectiveness of NaSIPA to dope PANI is much smaller and approaches a plateau at acid concentrations $\sim 0.5 \mathrm{M}$ (Fig. 3). The tendency of NaSIPA to form dimers, trimers or tetramers in aqueous solutions account for the low protonation degree $(\mathrm{S} / \mathrm{N}=0.24 \pm 0.03)[27]$.

Doping levels are further confirmed by the shift of the benzenoid (B) band in the FTIR spectra (Fig S4, S5 and S6). Furthermore, the relative intensity between the quinoid (Q) and benzenoid $(\mathrm{B})$ bands $\left(\mathrm{I}_{\mathrm{Q} / \mathrm{B}}\right)$ provides information on the oxidation level of the polymer. The ratio is closest to the emeraldine form $\left(\mathrm{I}_{\mathrm{Q} / \mathrm{B}}=1\right)$ in PANI-DBSA $0.1 \mathrm{M}$ $\left(\mathrm{I}_{\mathrm{Q} / \mathrm{B}}=0.93\right)$. Minor decreases in the intrinsic oxidation state occurred with rising DBSA concentration, although there are no significant differences between $0.25 \mathrm{M}$ and $1.5 \mathrm{M}$ samples, except for PANI-DBSA 1.25 M, which shows a noteworthy reduction down to $\mathrm{I}_{\mathrm{Q} / \mathrm{B}}=0.75$. These values are coherent with the common knowledge that the quinoid imines are preferentially protonated in the protonic acid doping [28]. Quite the opposite, 
no variations are observed in the oxidation level of PANI-NaSIPA samples within experimental error $\left(\mathrm{I}_{\mathrm{Q} / \mathrm{B}}=0.91 \pm 0.01\right)$, which are also close to the emeraldine base.

In parallel with these modifications, shifts and increasing intensity of the bands at 1240 $\mathrm{cm}^{-1}\left(v_{\mathrm{C}-\mathrm{N} \cdot+}\right.$ in the polaron lattice $/ v_{\mathrm{C}-\mathrm{N}}$ in $\mathrm{BBB}$ unit) and $\sim 1129 \mathrm{~cm}^{-1}\left(\mathrm{Q}=\mathrm{NH}^{+}-\mathrm{B}\right.$ or B$\mathrm{NH}^{+}-\mathrm{B} / \delta_{\mathrm{C}-\mathrm{H}}$ ) are observed in the spectra of all PANI salts (Fig. S4 and S5). The intensities of the latter two bands, which reflect the concentration of polaron, bipolaron and electron delocalization [29] gradually increase with doping level, although relative variations are smaller in PANI-NaSIPA than in PANI-DBSA samples. Finally, a sharp raise in both bands is observed in the PANI-DBSA $1.25 \mathrm{M}$ spectrum, which is consistent with the maximum doping level and electrical conductivity, due to formation of the most delocalized structure.

Together with the higher doping level, the enhanced electrical conductivity of PANIDBSA with respect to PANI-NaSIPA is supported by structural differences observed by XRD diffraction and TEM.

Both PANIs have very low crystallinity degree (Tables S1 and S2), but the PANIDBSA diffraction patterns show an additional peak at small angle $\left(2 \theta=2.88^{\circ}\right.$ to $\left.3.19^{\circ}\right)$ (Fig S7) indicative of a layered mesomorphic structure, in which the alkyl tails of the counterions function as spacers with some degree of interdigitation between parallel planes of the polymer backbones. For a given dopant, both the intensity of the low angle reflection and the interlayer d-spacing are influenced by the doping level [30], as depicted in Fig S8A and B. Moreover, reductions in the interlayer spacing are followed by a shrinking in the $\pi-\pi$ interchain stacking distance between the PANI phenyl rings and viceversa. Accordingly, the maximum peak intensity and the shortest intralayer dspacing (better $\pi-\pi$ overlap) correspond to PANI-DBSA 1.25 M ( $\mathrm{S} / \mathrm{N}=0.75)$ (Table $\mathrm{S} 1$ ). Contrary to previous authors, the electrical conductivity of PANI-DBSA still increases for protonation levels $>50 \%$ despite the formation of bipolarons, which have restricted carrier mobility compared to polarons [31]. In our case, the improvement of electric properties at high doping level is assisted by the formation of the layered structure as opposed to a three-dimensional close packing unit-cell. Larger intensity of the lower angle peaks reflects an increase in the overall hydrocarbon order within the long alkyl side DBSA chains arranged between the layers, perpendicular to the PANI main chain. Simultaneously, the rigidity of the PANI chains is increased due to electronic repulsion between the hydrophobic alkyl DBSA chains and the polar nature of cationic PANI. 
This leads to a more planar chain conformation, with reduced torsion angles between the phenyl rings and the plane of the backbone, which favors $\pi-\pi$ stacking (interchain diffusion) and the effective conjugation length (intrachain transfer) [30, 32-34]. Therefore, both the interchain diffusion of carriers and the intrachain transportation are promoted (Fig 4). These data are consistent with improved carrier transport and a longer mean free path [12].

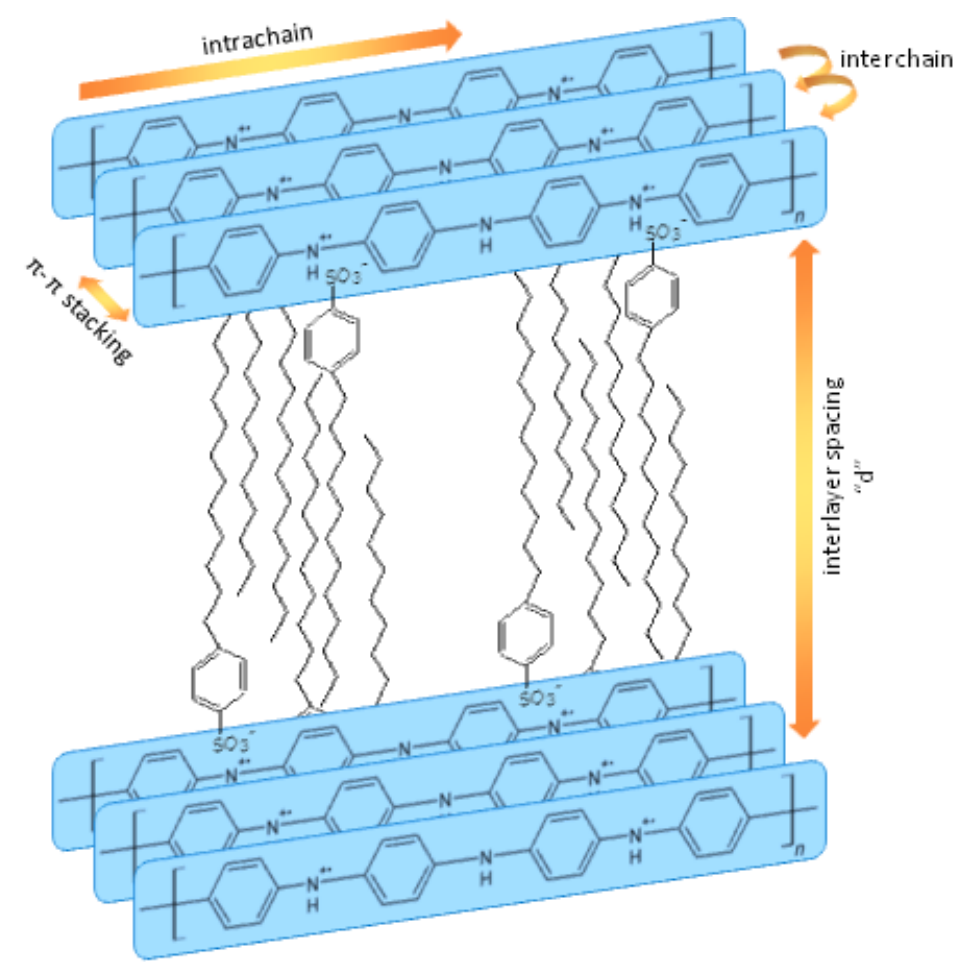

Figure 4. Schematic drawing proposed for the layered structure of PANI-DBSA.

The aforementioned structural differences between the two series of doped PANIs are also reflected in the morphology observed by TEM (Fig 5).
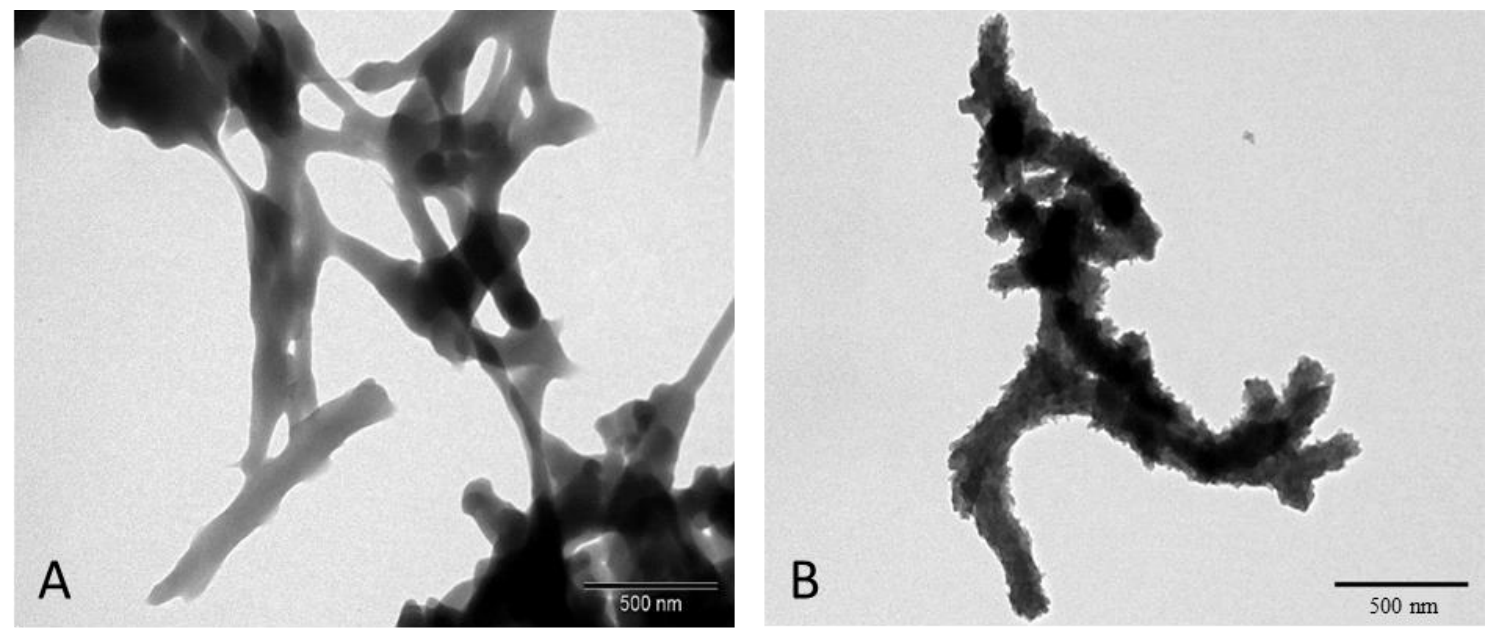
Figure 5. TEM images of A) PANI-DBSA 1.25M_50Kx and B) PANI-NaSIPA 1.25M_50Kx.

Nanorod structures, partially or totally covered by the agglomeration and adsorption of granules and flakes on their surface, are observed for low DBSA doping levels and all PANI-NaSIPA samples. For high DBSA molar concentrations, and due to its surfactant nature, these agglomerates evolve to a smooth enveloping layer, with no identifiable particle structures. This layer of chemically bound and free DBSA, forms a continuous network in compression-molded samples and may be a determining factor in the formation of conductive pathway structures [35, 36].

In summary, the higher electrical conductivity values of PANI-DBSA, in comparison with those of PANI-NaSIPA, are due to both the higher doping levels and the layer structure of PANI-DBSA.

\subsection{SEEBECK COEFFICIENT}

Fig 6 shows the variation of the Seebeck coefficient for the two PANI salts at room temperature as a function of the dopant molar concentration. Consistent with its high doping level, the Seebeck coefficient of PANI-DBSA is very small and fairly constant of the order of $1 \mu \mathrm{V} / \mathrm{K}$, within experimental error. Quite the opposite, the Seebeck coefficient of PANI - NaSIPA is between 4 and 34 times greater than that of PANIDBSA and shows a clear decrease upon raising the acid molar concentration up to NaSIPA $\approx 0.8 \mathrm{M}$. The last behaviour has been described previously by several authors $[1,37]$. Accordingly, the lightly doped PANI-NaSIPA samples have lower electrical conductivities and larger Seebeck coefficients compared to those of heavily doped PANI-DBSA[4]. 


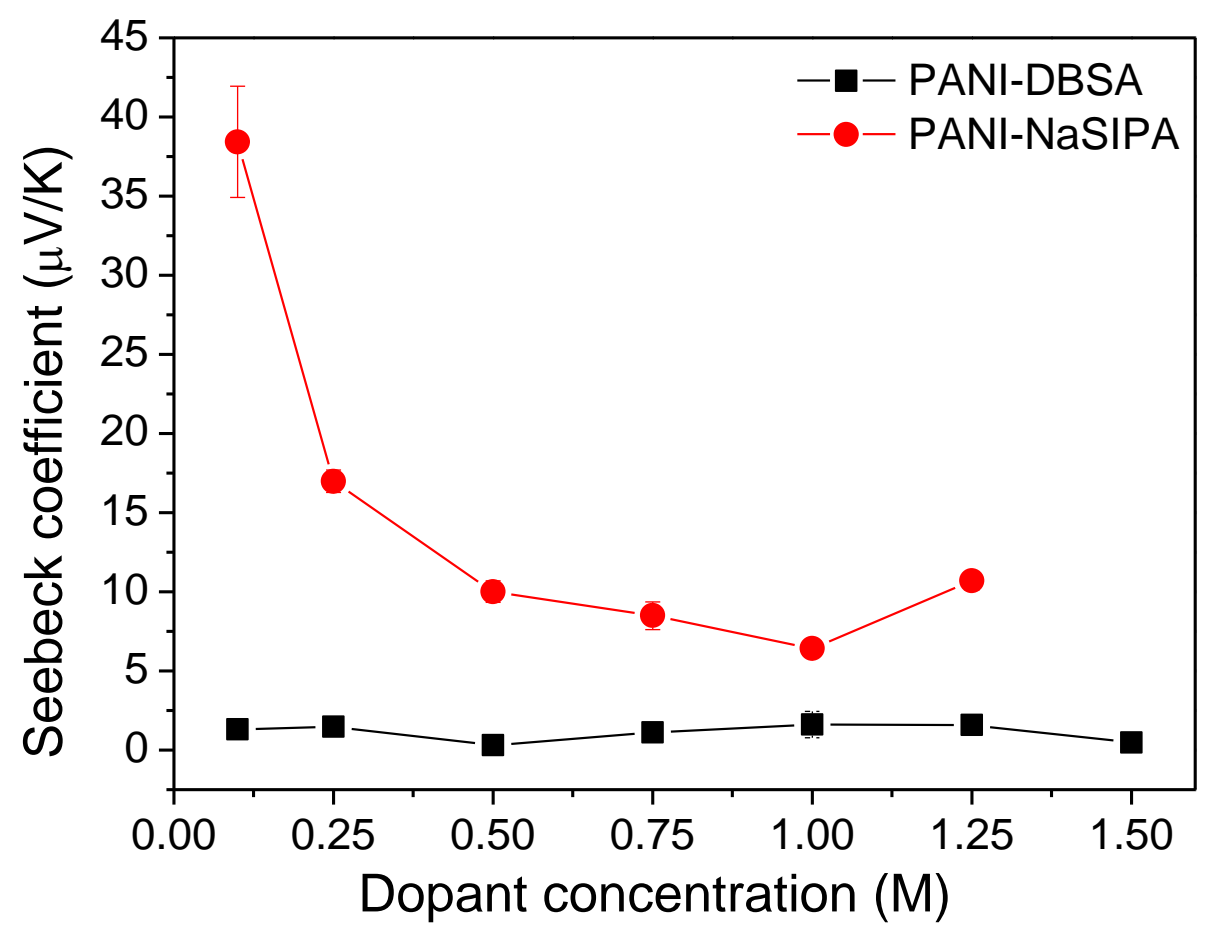

Figure 6. Seebeck coefficients of PANI-DBSA and PANI-NaSIPA at various molar concentrations at room temperature.

For both types of PANIs, Power Factors $\left(\mathrm{PF}=\alpha^{2} . \sigma\right)$ are of the order of $10^{-3} \mu \mathrm{Wm}^{-1} \mathrm{~K}^{-2}$. PANI-DBSA $1 \mathrm{M}$ and PANI-NaSIPA 0.5 M present an optimal compromise between the two properties involved; hence, these two samples, which have the same PF ( 4 $\mathrm{x}$ $10^{-3} \mu \mathrm{Wm}^{-1} \mathrm{~K}^{-2}$ ), have been chosen to study the temperature dependence of the TE properties.

The Seebeck coefficient is a very useful property to identify the intrinsic conduction processes in conducting polymers. As no current flows through the sample, it is not as dependent on material imperfections as electrical conductivity [38]. The temperature dependence of the Seebeck coefficient for samples of PANI-DBSA and PANI-NaSIPA is shown in Figure 7. 


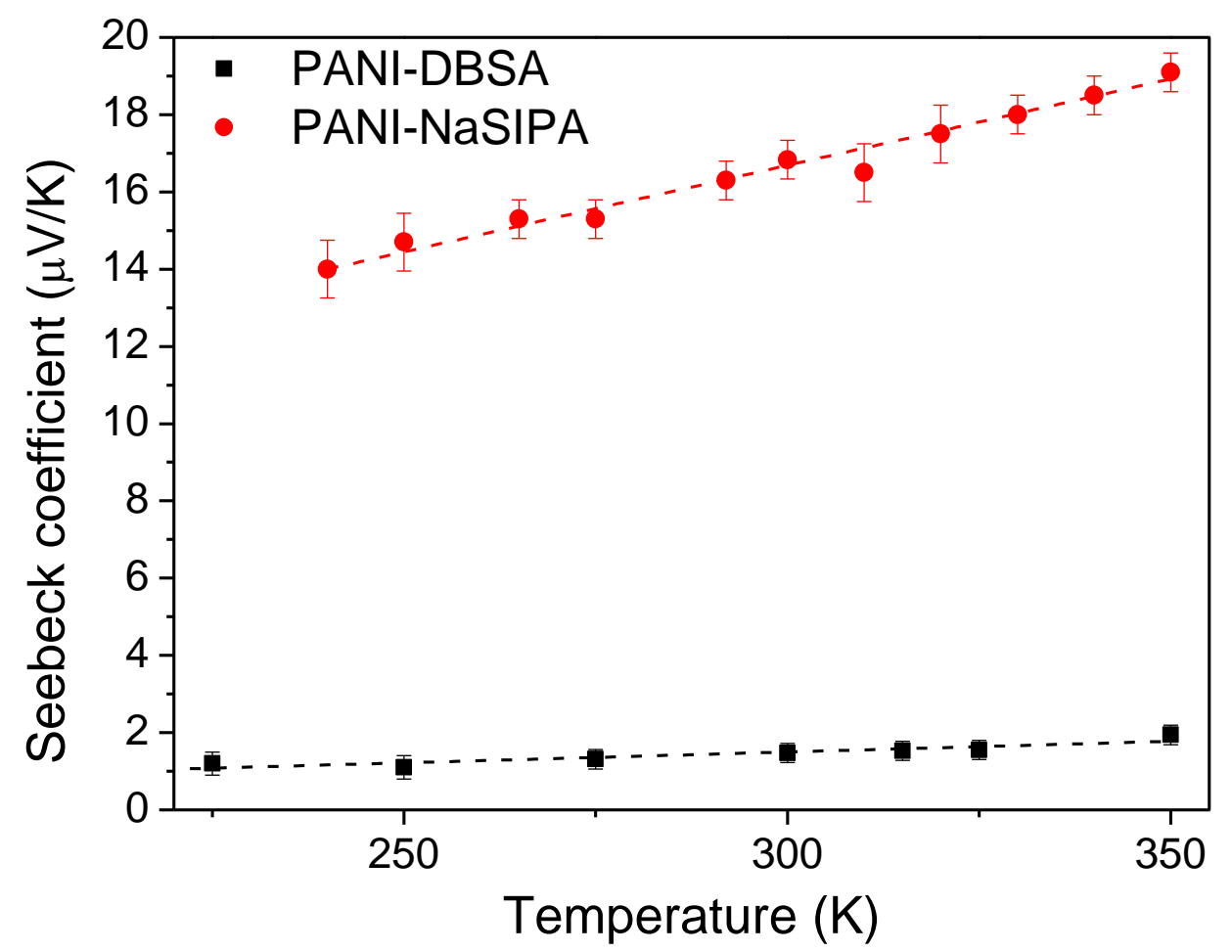

Figure 7. Temperature dependence of the Seebeck coefficient of PANI-DBSA $1 \mathrm{M}$ and PANI-NaSIPA $0.5 \mathrm{M}$.

The Seebeck coefficient of PANI-DBSA $1 \mathrm{M}$ is very small and nearly independent of temperature. This is characteristic of polaronic hopping, where $\alpha$ can be described from the Heike's formula:

$$
\alpha=\frac{k}{q} \ln \left(\frac{c}{1-c}\right)
$$

Here $c$ is the carrier concentration or doping level (carriers per site) and $q$ is the electron charge [4]. Fitting of the experimental data gives $c \approx 0.49$ (hole type), which is a little bit lower than the doping level estimated by the $\mathrm{S} / \mathrm{N}$ ratio $(0.65)$.

Concerning PANI-NaSIPA, although the conductivity is small and we could therefore expect an activated temperature dependence of the thermopower, it increases linearly with temperature in a wide temperature range, which is characteristic of diffusive (metallic-like) conduction. This apparent discrepancy probably reflects the inhomogeneous nature of the samples, with different transport mechanisms. Kaiser analysed the temperature dependence of the thermopower in heterogeneous conducting polymers (conducting fibrillar paths of polymer connected by regions with lower conductivity) with different dopant concentrations [39]. In this case, thermopower is 
dominated by the highly conducting regions, with a contribution $\propto \mathrm{T}^{1 / 2}$ from variable range hopping:

$$
\alpha=a T+b T^{1 / 2}
$$

For highly conducting samples the first term of this equation can be equated to the Mott's formula for thermopower [40].

If we assume a single parabolic band in the more conduction regions, the first term of equation (5) can be approximated by:

$$
\alpha=\frac{8 \pi^{2}}{3 e}\left(\frac{k_{B}}{h}\right)^{2} m^{*}\left(\frac{\pi}{3 n}\right)^{2 / 3} T
$$

Lee et al. measured an effective mass, $\mathrm{m}^{*} \approx 2 \mathrm{~m}_{\mathrm{e}}$ for highly conducting PANI [12]. Using this value in equation 6 , the estimated charge carrier density $n$ is $\approx 3 \times 10^{21} \mathrm{e} / \mathrm{cm}^{3}$ for PANI-NaSIPA. This result is consistent with that obtained from previous authors for this class of materials.

The observed behaviors for PANI-DBSA and PANI-NaSIPA differ from conclusions extracted from previous research works for homogeneous systems, where the Seebeck coefficient of lightly doped organic semiconductors is for the most part independent of temperature. Certainly, this is a complex issue and apart from the doping level, other factors, such as the degree of heterogeneity and order in the microstructure, must be taken into account and deserve further systematic exploration.

\subsection{THERMAL CONDUCTIVITY}

Regarding thermal conductivity, Figure 8 shows the variation of this parameter with the molar acid concentration for the two organic dopants at 300, 325 and $350 \mathrm{~K}$ [3, 41]. For PANI-DBSA samples, the thermal conductivity $(\kappa)$ is low and nearly constant $(0.14 \pm 0.2$ $\mathrm{W} / \mathrm{m} \cdot \mathrm{K}$ ) for DBSA molar concentrations below $0.5 \mathrm{M}$, then increases with the doping level up to $0.59 \pm 0.08 \mathrm{~W} / \mathrm{m} \cdot \mathrm{K}$ at room temperature. Quite the opposite, the thermal conductivity of PANI-NaSIPA is independent of the charge carriers within experimental error $(0.22 \pm 0.02 \mathrm{~W} / \mathrm{m} \cdot \mathrm{K})$. For both type of PANIs, and like most low crystalline polymers, the thermal conductivity increases with temperature $[4,8]$. The results lie within the range found in the literature for PANI thermal conductivity, spanning from 0.02 to $0.542 \mathrm{~W} . \mathrm{m}^{-1} \mathrm{~K}^{-1}$ [5]. Specifically, thermal conductivity values of $\sim 0.2 \mathrm{~W} . \mathrm{m}^{-1}$ $\mathrm{K}^{-1}$ have been reported for PANI-DBSA at $300 \mathrm{~K}$ [2]. 

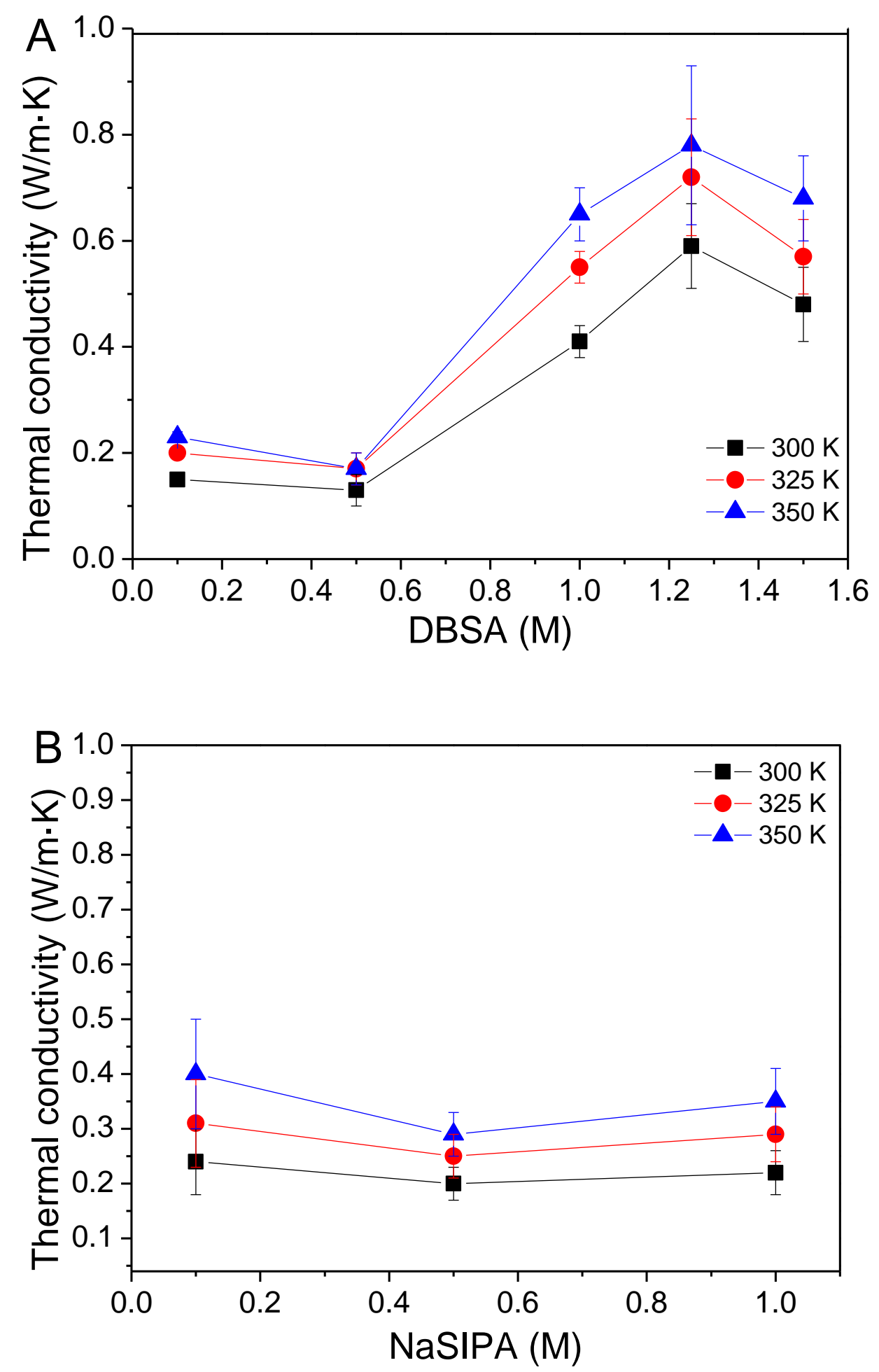

Figure 8. Thermal conductivity of A) PANI-DBSA B) PANI-NaSIPA at 300, 325 and $350 \mathrm{~K}$.

These low $\kappa$ values and the absence of correlation of the thermal conductivity with electrical conductivity are due to the van der Waals or H-bonding contact between molecules and structural disorder, that cause strong phonon scattering and negligible 
contribution of electrical carriers [42]. The thermal conductivity is the summation of an electronic part $\left(\kappa_{\mathrm{e}}\right)$ and a lattice contribution ( $\kappa_{\mathrm{ph}}$, phonons). A first approximation to the electronic contribution could be made from Wiedemann-Franz (WF) law:

$$
\kappa_{e}=L T \sigma
$$

where $\sigma$ is the electrical conductivity, $\mathrm{T}$ is the temperature and $\mathrm{L}$ is the Lorentz factor $\left(\mathrm{L}=2.45 \times 10^{-8} \mathrm{~W} \Omega \mathrm{K}^{-2}\right.$ ). Following Eq. (7), we have calculated a maximum value of $\kappa_{e}$ of the order of $10^{-6}$ to $10^{-4} \mathrm{~W} / \mathrm{m} \cdot \mathrm{K}$ at $300 \mathrm{~K}$. Although applying WF law to the conduction regime of a system like that studied here is a very crude approximation, it can give an idea of the order of magnitude of the electrical contribution to the thermal conductivity. Therefore, in accordance with previous studies on PANI [3, 43], we can safely assume that the total thermal conductivity is dominated by the lattice vibrations, which can be affected by the type and dopant concentration that may alter the heat capacity and the density of the polymer [4].

Furthermore, in the case of PANI-DBSA there is an increase in the thermal conductivity with doping level at $\mathrm{S} / \mathrm{N}$ values $>0.5$. These experimental findings may be ascribed to the formation of the layered structure, described in preceding paragraphs. Both the intensity of the low angle reflection and the interlayer d-spacing, increase at high doping levels (Fig. S7A); this morphology can decrease the boundary phonon scattering and promote the transport of phonons in the heat transfer process.

\section{4. $\quad$ FIGURE OF MERIT}

As a final point, the magnitude of the dimensionless thermoelectric figure of merit, ZT, determines the efficiency of the energy conversion. The ZT values of PANI-DBSA and PANI-NaSIPA, shown in Figure 9, are of the same order of magnitude than those found in the literature for pure bulk PANI at room temperature, which span from $7 \times 10^{-7}[44$, 45] to $\sim 2.75 \times 10^{-5}[8,37]$. In particular, a ZT of $\sim 1.5 \times 10^{-5}$ has been encountered for PANI-DBSA prepared by direct synthesis [2], whereas, as far as we know the thermoelectric properties of PANI-NaSIPA have not yet been investigated.

ZT of PANI-DBSA reaches its maximum values, $3.3 \times 10^{-6}$ and $3.2 \times 10^{-6}$, at high doping levels for DBSA 1 and 1.25M, respectively. Despite the higher electrical conductivity of PANI-DBSA $1.25 \mathrm{M}$, the simultaneous raise of the thermal conductivity, while the Seebeck coefficient remains nearly constant, explains the analogous value in relation with PANI-DBSA 1M. On the contrary for PANI-NaSIPA, ZT linearly decreases from 
NaSIPA $0.1 \mathrm{M}\left(1.0 \times 10^{-5}\right)$ to NaSIPA $1 \mathrm{M}\left(2.3 \times 10^{-6}\right)$, as a result of variations of the Seebeck coefficient and electrical conductivity. Hence, the ZT value of PANI-NaSIPA $0.5 \mathrm{M}$ is two orders of magnitude higher than that of PANI-DBSA $0.5 \mathrm{M}$, whereas similar ZT values are obtained at $1 \mathrm{M}$ acid concentration. Therefore, based on the variation trends of $\mathrm{ZT}$ with molar acid concentration, the present study offers the novel possibility of improving ZT of organic thermoelectric materials focusing on lightly doped PANIs.

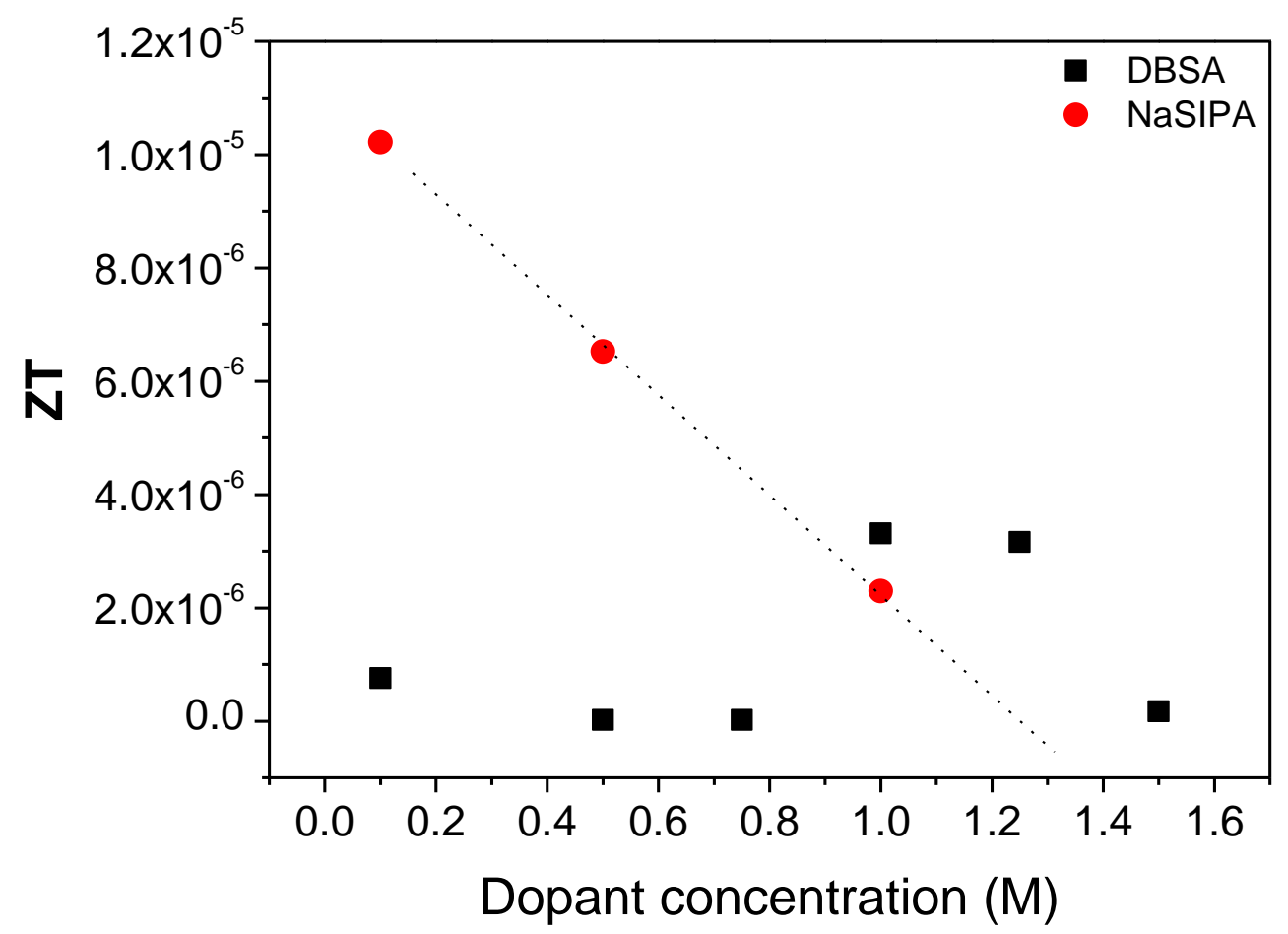

Figure 9. ZT values of PANI-DBSA and PANI-NaSIPA at different acid molar concentrations and $300 \mathrm{~K}$.

\section{CONCLUSIONS}

This study thoroughly describes the characterization of PANI nanorods prepared by an indirect synthetic route and doped with two organosulfonic acids, DBSA and NaSIPA at increasing molar acid concentrations. Carefully choosing the doping agent and adjusting the doping level provide conducting polymers with different structural order, morphology and TE efficiency that may be used for the fabrication of different performing devices for near room temperature applications.

PANI-DBSA materials need high doping levels, in conjunction with the formation of a well-defined layered structure, to achieve moderately high electrical conductivity and thus, maximize ZT, due to the low and constant Seebeck coefficient. The observed hopping behavior mechanism offers potential for thermal power generators with 
constant voltage that take advantage of the constancy of the Seebeck coefficient over a selected temperature range.

Opposite to this, NaSIPA displays poor doping ability due to its tendency to form dimers, trimers or tetramers in aqueous solutions; thus, the lightly doped PANI-NaSIPA samples show lower electrical conductivities and larger Seebeck coefficients than PANI-DBSA; both magnitudes show opposite trends upon raising the acid molar concentration. Notwithstanding, they have the advantage of moderately high ZT values at low acid molar concentrations, showing a diffusive regimen characteristic of degenerate metallic semiconductors from 200 to $350 \mathrm{~K}$.

\section{ACKNOWLEDGMENTS}

Authors acknowledge the financial support to Xunta de Galicia-FEDER (Program of Consolidation and structuring competitive research units (GRC2014/036)) and project MAT2013-44673-R.

\section{Data available}

The raw/processed data required to reproduce these findings cannot be shared at this time due to technical or time limitations.

\section{Supplementary data}

Description of the home-made device employed to measure the Seebeck coefficient and detailed discussion of the FTIR and X-ray diffraction data (PDF). This material is available free of charge via the Internet at

\section{REFERENCES}

[1] Q. Zhang, Y. Sun, W. Xu, D. Zhu, Organic Thermoelectric Materials: Emerging Green Energy Materials Converting Heat to Electricity Directly and Efficiently, Adv. Mater., 26 (2014) 6829-6851.

[2] W. Wang, S. Sun, S. Gu, H. Shen, Q. Zhang, J. Zhu, L. Wang, W. Jiang, One-pot fabrication and thermoelectric properties of $\mathrm{Ag}$ nanoparticles-polyaniline hybrid nanocomposites, RSC Advances, 4 (2014) 26810-26816.

[3] H. Yan, N. Sada, N. Toshima, Thermal transporting properties of electrically conductive polyaniline films as organic thermoelectric materials, J. Therm. Anal. Calorim., 69 (2002) 881-887. 
[4] O. Bubnova, X. Crispin, Towards polymer-based organic thermoelectric generators, Energy \& Environmental Science, 5 (2012) 9345-9362.

[5] Y. Du, S.Z. Shen, K. Cai, P.S. Casey, Research progress on polymer-inorganic thermoelectric nanocomposite materials, Prog. Polym. Sci., 37 (2012) 820-841.

[6] Z.U. Khan, J. Edberg, M.M. Hamedi, R. Gabrielsson, H. Granberg, L. Wågberg, I. Engquist, M. Berggren, X. Crispin, Thermoelectric Polymers and their Elastic Aerogels, Adv. Mater., 28 (2016) 4556-4562.

[7] B. Russ, A. Glaudell, J.J. Urban, M.L. Chabinyc, R.A. Segalman, Organic thermoelectric materials for energy harvesting and temperature control, Nature Reviews Materials, 1 (2016) 16050.

[8] J. Wu, Y. Sun, W. Xu, Q. Zhang, Investigating thermoelectric properties of doped polyaniline nanowires, Synth. Met., 189 (2014) 177-182.

[9] S. Bhadra, D. Khastgir, N.K. Singha, J.H. Lee, Progress in preparation, processing and applications of polyaniline, Prog. Polym. Sci., 34 (2009) 783-810.

[10] T.A. Skotheim, Handbook of conducting polymers, CRC press1997.

[11] S.D. George, S. Saravanan, M. Anantharaman, S. Venkatachalam, P. Radhakrishnan, V. Nampoori, C. Vallabhan, Thermal characterization of doped polyaniline and its composites with CoPc, Physical Review B, 69 (2004) 235201.

[12] K. Lee, S. Cho, S.H. Park, A. Heeger, C.-W. Lee, S.-H. Lee, Metallic transport in polyaniline, Nature, 441 (2006) 65-68.

[13] V. Prigodin, A. Epstein, Quantum hopping in metallic polymers, Physica B: Condensed Matter, 338 (2003) 310-317.

[14] O. Bubnova, Thermoelectric properties of conducting polymers, Linköping University Electronic Press, 2013.

[15] A.B. Kaiser, C.K. Subramaniam, P.W. Gilberd, B. Wessling, Electronic transport properties of conducting polymers and polymer blends, Synth. Met., 69 (1995) 197-200. [16] A.B. Kaiser, Systematic conductivity behavior in conducting polymers: effects of heterogeneous disorder, Adv. Mater., 13 (2001) 927-941.

[17] Y. Sun, Z. Wei, W. Xu, D. Zhu, A three-in-one improvement in thermoelectric properties of polyaniline brought by nanostructures, Synth. Met., 160 (2010) 23712376.

[18] L. Horta-Romarís, M.-J. Abad, M.V. González-Rodríguez, A. Lasagabáster, P. Costa, S. Lanceros-Méndez, Cyclic temperature dependence of electrical conductivity in 
polyanilines as a function of the dopant and synthesis method, Materials \& Design, 114 (2017) 288-296.

[19] M.S. Dopico-García, A. Ares, A. Lasagabáster-Latorre, X. García, L. Arboleda, M.J. Abad, Extruded polyaniline/EVA blends: Enhancing electrical conductivity using gallate compatibilizers, Synth. Met., 189 (2014) 193-202.

[20] P. Saini, R. Jalan, S.K. Dhawan, Synthesis and characterization of processable polyaniline doped with novel dopant NaSIPA, J. Appl. Polym. Sci., 108 (2008) 14371446.

[21] H.-W. Park, T. Kim, J. Huh, M. Kang, J.E. Lee, H. Yoon, Anisotropic Growth Control of Polyaniline Nanostructures and Their Morphology-Dependent Electrochemical Characteristics, ACS Nano, 6 (2012) 7624-7633.

[22] J. Yang, B. Weng, Inverse emulsion polymerization for high molecular weight and electrically conducting polyanilines, Synth. Met., 159 (2009) 2249-2252.

[23] M. Wojdyr, Fityk: a general-purpose peak fitting program, J. Appl. Crystallogr, 43 (2010) 1126-1128.

[24] J.P. Pouget, M.E. Jozefowicz, A.J. Epstein, X. Tang, A.G. MacDiarmid, X-ray structure of polyaniline, Macromolecules, 24 (1991) 779-789.

[25] P. Jayamurgan, V. Ponnuswamy, S. Ashokan, T. Mahalingam, The effect of dopant on structural, thermal and morphological properties of DBSA-doped polypyrrole, Iranian Polymer Journal, 22 (2013) 219-225.

[26] M.G. Han, S.K. Cho, S.G. Oh, S.S. Im, Preparation and characterization of polyaniline nanoparticles synthesized from DBSA micellar solution, Synth. Met., 126 (2002) 53-60.

[27] J.F. Duan, Sulfoisophthalic acid solution process therewith, Google Patents, 2002.

[28] N.A. Ogurtsov, Y.V. Noskov, V.N. Bliznyuk, V.G. Ilyin, J.-L. Wojkiewicz, E.A. Fedorenko, A.A. Pud, Evolution and interdependence of structure and properties of nanocomposites of multiwall carbon nanotubes with polyaniline, The Journal of Physical Chemistry C, 120 (2015) 230-242.

[29] M. Trchová, J. Stejskal, Polyaniline: the infrared spectroscopy of conducting polymer nanotubes (IUPAC Technical Report), Pure Appl. Chem., 83 (2011) 18031817.

[30] T. Taka, J. Laakso, K. Levon, Conductivity and structure of DBSA-protonated polyaniline, Solid state communications, 92 (1994) 393-396. 
[31] S. Bhadra, N.K. Singha, S. Chattopadhyay, D. Khastgir, Effect of different reaction parameters on the conductivity and dielectric properties of polyaniline synthesized electrochemically and modeling of conductivity against reaction parameters through regression analysis, J. Polym. Sci., Part B: Polym. Phys., 45 (2007) 2046-2059.

[32] W.-Y. Zheng, K. Levon, T. Taka, J. Laakso, J. Osterholm, Doping-induced layered structure in N-alkylated polyanilines, Polym. J., 28 (1996) 412-418.

[33] K. Levon, K.H. Ho, W.Y. Zheng, J. Laakso, T. Kärnä, T. Taka, J.E. Österholm, Thermal doping of polyaniline with dodecylbenzene sulfonic acid without auxiliary solvents, Polymer, 36 (1995) 2733-2738.

[34] M.G. Han, S.S. Im, Electrical and structural analysis of conductive polyaniline/polyimide blends, J. Appl. Polym. Sci., 71 (1999) 2169-2178.

[35] G.I. Titelman, A. Siegmann, M. Narkis, Y. Wei, Morphology of polyaniline redoped by kneading with dodecylbenzene sulfonic acid, J. Appl. Polym. Sci., 69 (1998) 2205-2212.

[36] Y. Roichman, G. Titelman, M. Silverstein, A. Siegmann, M. Narkis, Polyaniline synthesis: influence of powder morphology on conductivity of solution cast blends with polystyrene, Synth. Met., 98 (1999) 201-209.

[37] N. Mateeva, H. Niculescu, J. Schlenoff, L.R. Testardi, Correlation of Seebeck coefficient and electric conductivity in polyaniline and polypyrrole, J. Appl. Phys., 83 (1998) 3111-3117.

[38] A.B. Kaiser, Electronic transport properties of conducting polymers and carbon nanotubes, Rep. Prog. Phys., 64 (2001) 1.

[39] A. Kaiser, Thermoelectric power and conductivity of heterogeneous conducting polymers, Physical Review B, 40 (1989) 2806.

[40] N.F. Mott, H. Jones, The theory of the properties of metals and alloys, Courier Corporation 1958.

[41] M. Amrithesh, P.A. Francis Xavier, V.G. Chandraprabhu, S. Jayalekshmi, S.T. Lee, J. Ravi, Thermal diffusivity measurements in PANI and PANI-MWNT composites using photo acoustic technique, Trans Indian Inst Met, 64 (2011) 133-136.

[42] R.A. Schlitz, F.G. Brunetti, A.M. Glaudell, P.L. Miller, M.A. Brady, C.J. Takacs, C.J. Hawker, M.L. Chabinyc, Solubility-Limited Extrinsic n-Type Doping of a High Electron Mobility Polymer for Thermoelectric Applications, Adv. Mater., 26 (2014) 2825-2830. 
[43] C. Nath, A. Kumar, K.-Z. Syu, Y.-K. Kuo, Heat conduction in conducting polyaniline nanofibers, Appl. Phys. Lett., 103 (2013) 121905.

[44] Q. Yao, L. Chen, W. Zhang, S. Liufu, X. Chen, Enhanced Thermoelectric Performance of Single-Walled Carbon Nanotubes/Polyaniline Hybrid Nanocomposites, ACS Nano, 4 (2010) 2445-2451.

[45] P. Limelette, B. Schmaltz, D. Brault, M. Gouineau, C. Autret-Lambert, S. Roger, V. Grimal, F. Tran Van, Conductivity scaling and thermoelectric properties of polyaniline hydrochloride, J. Appl. Phys., 115 (2014) 033712. 